\title{
Standardisierte Gerinnungsanamnese vor Tonsillektomie und Adenotomie im Kindesalter
}

Boris A. Stuck ${ }^{1}$, Maren Königstein ${ }^{2}$, Claudia Umbreit ${ }^{2}$, Ute Walliczek ${ }^{3}$, Angela Wenzel ${ }^{2}$

${ }^{1}$ Klinik für Hals-Nasen-Ohren-Heilkunde, Kopf- und Hals-Chirurgie (Direktor: Prof. Dr. S. Lang), Universität Duisburg-Essen

2Universitäts-HNO-Klinik Mannheim (Direktor: Prof. Dr. med. K. Hörmann)

${ }^{3}$ Hals-Nasen-Ohren-Klinik (Direktor: Prof. Dr. med. J. A. Werner), Universitätsklinikum Gießen-Marburg, Standort Marburg

\section{Einleitung}

Im Jahr 2006 wurde ein Positionspapier zur präoperativen Gerinnungsdiagnostik bei Kindern vor einer Tonsillektomie Adenotomie (TE/AT) veröffentlicht [1] Dieses empfiehlt, auf die präoperative "Routinegerinnung" zu verzichten und stattdessen einen standardisierten Fragebogen zur Abklärung eine Blutungsneigung zu verwenden. Ziel der vorliegenden Studie war es zu überprüfen, ob es nach der Umsetzung de Empfehlung zu einer veränderten Nachblutungsrate gekommen ist.

\section{Material und Methoden}

Mittels einer retrospektiven Datenanalyse der Jahre 2003 und 2009 wurde die Inzidenz von Nachblutungen bei Kindern nach TE, AT und TE+AT untersucht Während 2003 nur eine freie Anamnese durchgeführt wurde und in Teilen eine Routinegerinnung vorlag, wurden 2009 alle Kinder durch den standardisierten Fragebogen (siehe Abb. 1) gescreent und im Verdachtsfall eine umfassende Gerinnungsdiagnostik vorgenommen.
Abbildung 1: Standardisierter Fragebogen zur Gerinnungsanamnese

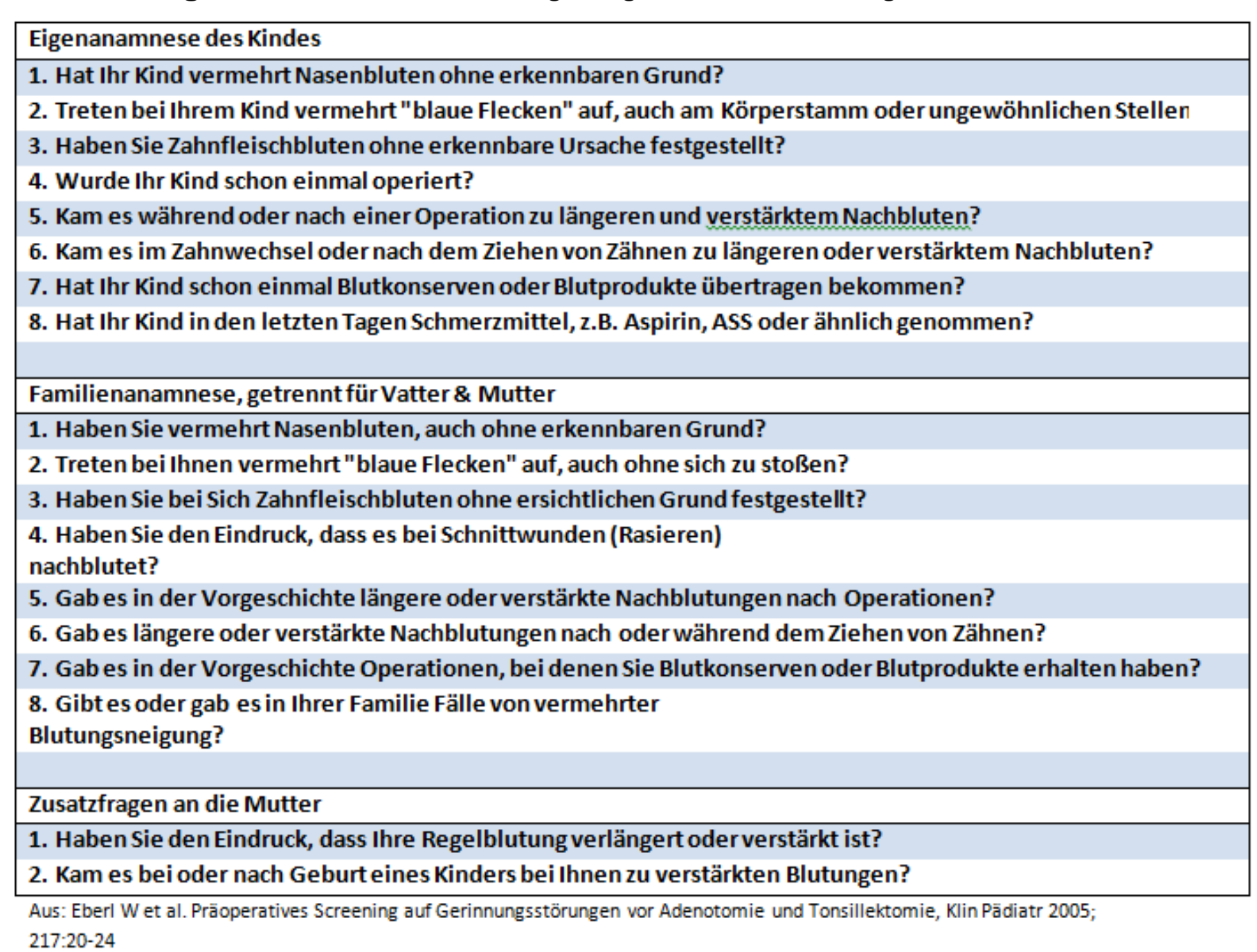

217:20-24

\section{Ergebnisse}

2003 wurden 293 Kinder operiert, in 20 Fällen (6.8\%) kam es zu Nachblutungen, wovon 18 (6\%) revidiert werden mussten. 2009 wurden 352 Kinder operiert, in 25 Kinder hatten nachgeblutet (7.1\%), 14 (4\%) wurden operativ revidiert. Eine klinisch relevante Änderung der Häufigkeit oder statistisch signifikante Unterschiede in den Nachblutungshäufigkeiten ergaben sich nicht (siehe Abb. 2). Bei 5 der Kinder, die 2003 nachgeblutet hatten, lag präoperativ ein Gerinnungslabor vor, das in allen Fällen unauffällig war. Auch alle postoperativ durchgeführten Gerinnungsuntersuchungen, die im Anschluss an die Nachblutungen in beiden Gruppen durchgeführt wurden, waren unauffällig.

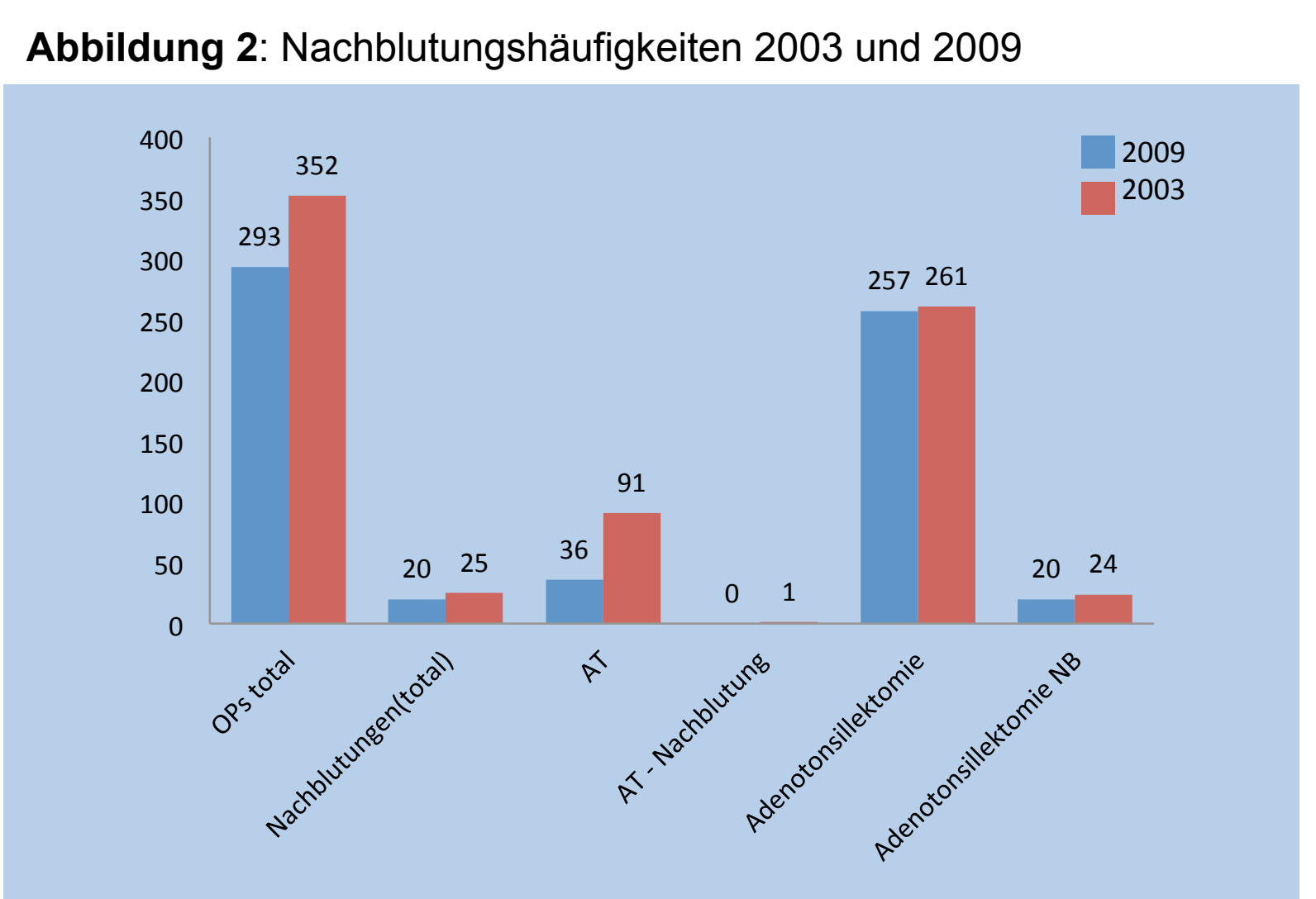

\section{Schlussfolgerung}

Die Anwendung des standardisierten Fragenbogens unter Verzicht auf eine Routinegerinnung führt nicht zu einer nachweisbaren Veränderung der Nachblutungsrate nach TE/AT. Die Ergebnisse bestätigen darüber hinaus, dass Nachblutungen nach TE/AT in aller Regel nicht Ausdruck einer kompromittierten Blutgerinnung sind.

\section{Literatur}

1. Hörmann K: Gemeinsame Stellungnahme zur Notwendigkeit präoperativer Gerinnungsdiagnostik vor Rhino-Otol 2006; 85: 580-1 